\title{
Use of routinely collected data in a UK cohort of publicly
}

\section{funded randomised clinical trials [version 1; peer review: 1}

\section{approved with reservations]}

\author{
Andrew J. McKay (D1), Ashley P. Jones', Carrol L. Gamble1,2, Andrew J. Farmer³, \\ Paula R. Williamson² \\ ${ }^{1}$ Liverpool Clinical Trials Centre, University of Liverpool, a member of Liverpool Health Partners, Liverpool, UK \\ ${ }^{2}$ MRC North West Hub for Trials Methodology Research, Department of Biostatistics, University of Liverpool, Liverpool, UK \\ ${ }^{3}$ Nuffield Department of Primary Care Health Sciences, University of Oxford, Oxford, UK
}

V1 First published: 04 May 2020, 9:323

https://doi.org/10.12688/f1000research.23316.1

Second version: 01 Jun 2020, 9:323

https://doi.org/10.12688/f1000research.23316.2

Latest published: 12 Mar 2021, 9:323

https://doi.org/10.12688/f1000research.23316.3

\section{Abstract}

Routinely collected data about health in medical records, registries and hospital activity statistics is now routinely collected in an electronic form. The extent to which such sources of data are now being routinely accessed to deliver efficient clinical trials, is unclear. The aim of this study was to ascertain current practice amongst a United Kingdom (UK) cohort of recently funded and ongoing randomised controlled trials (RCTs) in relation to sources and use of routinely collected outcome data.

Recently funded and ongoing RCTs were identified for inclusion by searching the National Institute for Health Research journals library. Trials that have a protocol available were assessed for inclusion and those that use or plan to use routinely collected health data for at least one outcome were included. Routinely collected data sources and outcome information were extracted.

A total of 279 studies were identified with 102 eligible for data extraction. An Electronic Health Record (EHR) was the sole source of outcome data for at least one outcome in 46 trials. The most frequent sources are Hospital Episode Statistics (HES) and Office for National Statistics (ONS), with the most common outcome data to be extracted being on mortality, hospital admission, and health service resource use.

Our study has found that around half of publicly funded trials in a UK cohort plan to collect outcome data from routinely collected data sources. This is much higher than the figure of $8 \%$ found in a cohort of 189 RCTs published since 2000, the majority of were carried out in North America (McCord et al., 2019).

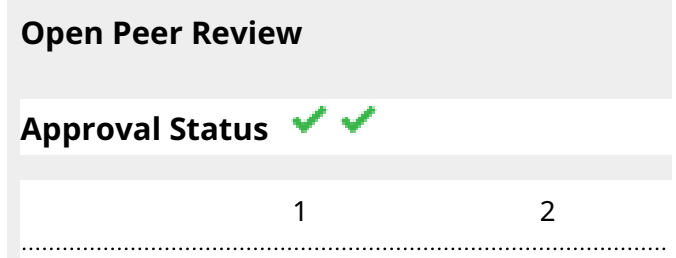

version 3

(revision)

12 Mar 2021

version 2

(revision)

01 Jun 2020

version 1

04 May 2020

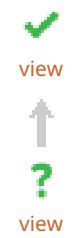

1. Sharon Love ID, University College London, London, UK

2. Alison Howie ID, University College London, London, UK

Merrick Zwarenstein, University College London, London, UK

Any reports and responses or comments on the article can be found at the end of the article. 


\section{Keywords}

Electronic Health Records, Data linkage, EHR, NIHR HTA, Randomised

Clinical Trial, Randomised Controlled Trial, RCT, Registry, Routinely

collected data

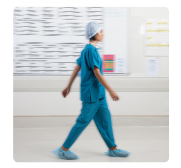

This article is included in the Health Services

gateway.

Corresponding author: Paula R. Williamson (p.r.williamson@liverpool.ac.uk)

Author roles: McKay AJ: Conceptualization, Data Curation, Formal Analysis, Investigation, Methodology, Project Administration, Resources, Validation, Visualization, Writing - Original Draft Preparation, Writing - Review \& Editing; Jones AP: Conceptualization, Investigation, Methodology, Project Administration, Resources, Visualization, Writing - Review \& Editing; Gamble CL: Conceptualization, Investigation, Methodology, Project Administration, Resources, Visualization, Writing - Review \& Editing; Farmer AJ: Conceptualization, Investigation, Methodology, Project Administration, Resources, Visualization, Writing - Review \& Editing; Williamson PR: Conceptualization, Data Curation, Formal Analysis, Investigation, Methodology, Project Administration, Resources, Supervision, Validation, Visualization, Writing - Original Draft Preparation, Writing - Review \& Editing

Competing interests: AJF is Chair of an NIHR HTA Funding Committee. CLG is Director of the Liverpool Clinical Trials Centre which receives NIHR Clinical Trials Unit support funding.

Grant information: AJF is an NIHR Senior Investigator and receives support from NIHR Oxford Biomedical Research Centre. PRW is an NIHR Senior Investigator and lead for the MRC/NIHR Trials Methodology Research Partnership (Grant reference: MR/S014357/1). The funders had no role in study design, data collection and analysis, decision to publish, or preparation of the manuscript.

Copyright: (c) 2020 McKay AJ et al. This is an open access article distributed under the terms of the Creative Commons Attribution License , which permits unrestricted use, distribution, and reproduction in any medium, provided the original work is properly cited.

How to cite this article: McKay AJ, Jones AP, Gamble CL et al. Use of routinely collected data in a UK cohort of publicly funded randomised clinical trials [version 1; peer review: 1 approved with reservations] F1000Research 2020, 9:323

https://doi.org/10.12688/f1000research.23316.1

First published: 04 May 2020, 9:323 https://doi.org/10.12688/f1000research.23316.1 


\section{Introduction}

Routinely collected data about health in medical records, registries and hospital activity statistics is now routinely collected in an electronic form. Progress in achieving connectivity, data linkage and security now offers the possibility of better use of this data for research purposes. For example, recent evidence shows the utility of long-term follow-up of trial patients through the electronic health record (EHR) (Fitzpatrick et al., 2018). Innovative data-enabled study designs can answer pressing knowledge gaps in research evidence. However, the extent to which such sources of data are now being routinely employed in research to deliver efficient clinical trials, potentially at a wide scale, is unclear.

The aim of this study was to ascertain current practice amongst a United Kingdom (UK) cohort of recently funded and ongoing randomised controlled trials (RCTs) in relation to sources and use of routinely collected outcome data. We define routinely collected health data to be data collected without specific a priori research questions developed prior to using the data for research.

\section{Methods}

Inclusion criteria

The following inclusion criteria were used:

1. Ongoing RCT of any type including feasibility or pilot work, funded by the National Institute for Health Research (NIHR) Health Technology Assessment (HTA) programme;

2. use of routinely collected health data for at least one study outcome; and

3. availability of a protocol.

\section{Search methods}

A search of the NIHR Journals Library was undertaken to find protocols registered as of 25/10/2019. The search fields and terms used to select were:

1. Search term: 'Random'

2. Research type: 'Primary research'

3. Programme: 'HTA'

4. Status: 'Research in progress'

If the final published report was shown alongside the protocol this was taken to mean that the RCT was not ongoing but the status had not been updated to 'Published', and the study was excluded.

In the absence of a protocol, the study was excluded. For studies with multiple protocol versions, the most recently available version was used.

\section{Data extraction}

One person (AM) extracted the information and categorised each EHR, with a second person (PW) checking classifications and explanations. The information extracted was as follows: Lead Investigator surname, year started, ISRCTN, project title, study type, use of routinely collected health data for at least one study outcome, availability of a protocol, any details of EHR data quality assessment prior to use, EHR name, reasons for sourcing outcome data from EHR, specific outcomes and outcome type where clear data to be used will come from named EHRs.

\section{Results}

Figure 1 shows the study flow diagram. There were 102 eligible trials available for further study.

Table 1 shows the reasons for collecting trial outcome data from routine sources. The EHR was the sole source of outcome data for at least one outcome in 46 trials (categories 3, 4 and 6 in Table 1). In five of these 46 protocols there was reference to prior feasibility work confirming aspects of the quality of the data to be sufficient for the main trial. Of the 102 trials, 14 (categories $7 \mathrm{a}-7 \mathrm{~d}$ in Table 1) planned to assess the feasibility of using the EHR data sources during the trial, although details of the assessment were often lacking. Raw data for Figure 1 and Table 1 and Table 2 are available (see Underlying data, McKay et al. (2020)).

Table 2 shows the sources of outcome data to be used in these 46 studies. The most frequent sources are Hospital Episode Statistics (HES) and Office for National Statistics (ONS), with the most common outcome data to be extracted being on mortality, hospital admission, and health service resource use (see Underlying data, Data Set 5; McKay et al. (2020)). The full list of data sources is given in Extended data, Supplementary Table 1 (McKay et al., 2020).

\section{Discussion}

Our study has found that around half of publicly funded trials in a UK cohort plan to collect outcome data from routinely collected data sources. This is much higher than the figure of $8 \%$ found in a cohort of 189 RCTs published since 2000, the majority of which were carried out in North America (McCord et al., 2019).

Very few trial teams described any assessments of data quality from EHRs in the protocol. Work is ongoing that should determine whether such information should be reported in the trial publication (Kwakkenbos et al., 2018). An extension to the SPIRIT guidelines for EHR-supported trials is soon to be initiated, and will determine whether this information should be included in the trial protocol. As a minimum, it is recommended that trialists provide evidence in any funding application about the quality of the data from the EHR. 


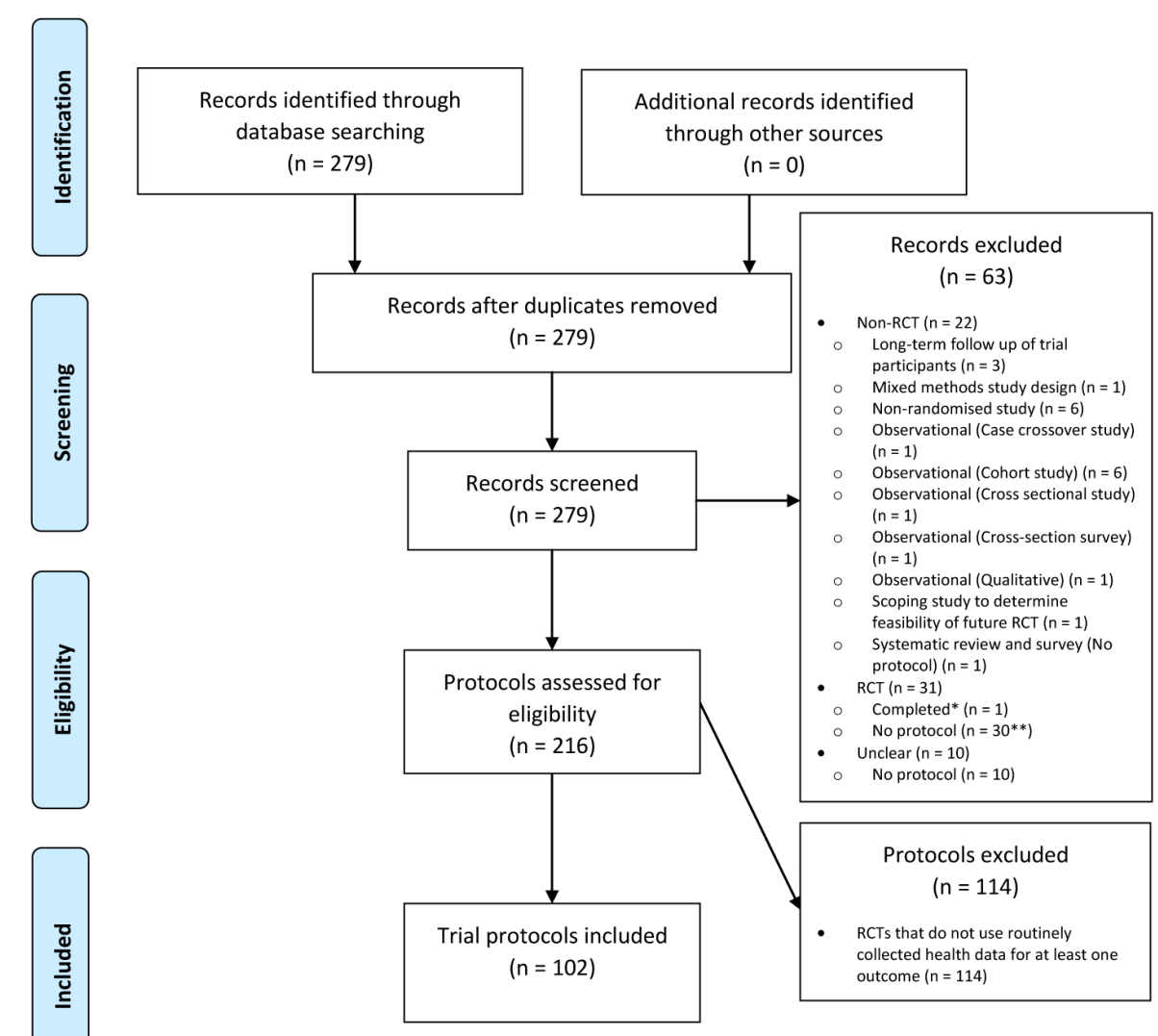

* Within NIHR website the research status had not been manually updated to be 'Completed research' so was still classed as 'Ongoing research' at the time of searching

** No protocols were available online for trials started in the following years: $2010(n=1), 2013$ ( $n=1$ ), 2017 ( $n=2), 2018$ (n=9), 2019 ( $n=17)$

Figure 1. PRISMA flow diagram.

Table 1. Reasons for sourcing outcome data from EHRs in 102 studies. Multiple categories can apply to a single study.

\section{Categories}

(1) (1a) 'Supplementing data collection for withdrawn patients (consent asked for at time of withdrawal)'

(1b) 'Supplementing data collection for lost-to-follow-up patients'

(1c) 'Supplementing data collection for withdrawn patients (consent NOT ASKED FOR at time of withdrawal)'

(1e) 'Continued data collection for withdrawn patients (consent asked for at time of withdrawal)'

(2) (2) 'Supplementing data collection for unobtainable/missing data'

(3) (3a) 'As the sole source of all outcome data'

(3b) 'As the sole source of all outcome data except for data related to protocol adherence and adverse event reporting being collected using CRFs'

(4) (4) 'As the sole source of some outcome data'

(5) (5a) 'As a source of some outcome data, alongside other sources for the same outcome data (e.g. CRF)'

(5b) 'As a source of some outcome data, but collected by CRF if unable to access data'

(6) (6a) 'Registry trial: As the sole source of outcome data with purpose-built Module to collect remaining outcome data'

(6b) 'Registry trial: All outcome data collected through multiple EHRs except for questionnaire data'

(6c) 'Registry trial: All outcome data collected through multiple EHRs except for some baseline data, questionnaire data and other patient-reported data' 


\begin{tabular}{|c|c|c|}
\hline & Categories & Total \\
\hline \multirow[t]{5}{*}{ (7) } & (7a) 'EHR data compared to trial collected data as part of feasibility assessment criteria' & 11 \\
\hline & (7b) 'EHR data compared to trial collected data as a main trial secondary outcome' & 1 \\
\hline & (7c) 'EHR data compared to trial collected data and then collect long-term follow-up data as part of trial' & 1 \\
\hline & $\begin{array}{l}\text { (7d) 'EHR data compared to trial collected data and then collect long-term follow-up data after trial has been } \\
\text { completed' }\end{array}$ & 1 \\
\hline & $\begin{array}{l}\text { (7e) 'Representativeness of randomised patients compared with all eligible patients using EHR data as part } \\
\text { of feasibility assessment criteria' }\end{array}$ & 1 \\
\hline \multirow[t]{2}{*}{ (8) } & $\begin{array}{l}\text { (8a) 'Participants flagged with NHS Digital/other: Check health status of patient prior to contacting in case } \\
\text { patient has died' }\end{array}$ & 2 \\
\hline & (8b) 'Participants flagged with NHS Digital/other: Check health status/notification of any deaths, causes' & 12 \\
\hline (9) & (9) 'Set up mechanisms for long-term follow-up' & 4 \\
\hline \multirow[t]{2}{*}{ (10) } & $\begin{array}{l}\text { (10) 'Patients asked to provide written consent for continuation in the study once have regained capacity. } \\
\text { Those who prefer not to be actively involved in the study follow-up, then asked to provide co }\end{array}$ & 1 \\
\hline & Total & 155 \\
\hline
\end{tabular}

\section{Table 2. Categories of EHR sources of outcome data in 46 studies where this was the sole source for at least one outcome.}

\begin{tabular}{|l|l|}
\hline Source & Number (\%) \\
\hline (i) Primary care data (all regional equivalents) & $8(17 \%)$ \\
\hline (ii) HES (and/or regional equivalents) & $27(59 \%)$ \\
\hline $\begin{array}{l}\text { (iii) ONS (and/or regional equivalents) } \\
\begin{array}{l}\text { (iv) Data collected specifically for patient group } \\
\text { or healthcare intervention (to include patient } \\
\text { registries, ICNARC, ambulance, etc) }\end{array}\end{array}$ & $27(59 \%)$ \\
\hline \begin{tabular}{l} 
(v) Other \\
\hline
\end{tabular} & $5(11 \%)$ \\
\hline
\end{tabular}

\section{Data availability}

Underlying data

Figshare: Use of routinely collected data in a UK cohort of publicly funded randomised clinical trials. https://doi.org/10.6084/ m9.figshare.12185193 (McKay et al., 2020).

This project contains the following underlying data:

- Data_Set_1_Details_and_Figure_1_v1.0.csv. (Study identifiers and raw data used for Figure 1.)
- Data_Set_2_Table_1_v1.0.csv. (Raw data used for Table 1.)

- Data_set_3_Supp_Table_1_v1.0.csv. (Raw data used for Supplementary Table 1.)

- Data_set_4_Table_2_v1.0.csv. (Raw data used for Table 2.)

- Data_set_5_Outcomes_using_EHR_data_v1.0.csv. (Raw data showing details of outcomes using EHR data.)

\section{Extended data}

Figshare: Use of routinely collected data in a UK cohort of publicly funded randomised clinical trials. https://doi.org/10.6084/ m9.figshare.12185193 (McKay et al., 2020).

This project contains the following extended data:

- Supplementary Table 1 - EHR sources of outcome data v1.0.pdf. (Supplementary Table 1.)

Data are available under the terms of the Creative Commons Zero "No rights reserved" data waiver (CCO 1.0 Public domain dedication).
Fitzpatrick T, Perrier L, Shakik S, et al: Assessment of Long-term Follow-up of Randomized Trial Participants by Linkage to Routinely Collected Data: A Scoping Review and Analysis. JAMA Netw Open. 2018; 1(8): e186019. PubMed Abstract | Publisher Full Text | Free Full Text

Kwakkenbos L, Juszczak E, Hemkens LG, et al.: Protocol for the development of a CONSORT extension for RCTs using cohorts and routinely collected health data. Res Integr Peer Rev. 2018; 3: 9.

PubMed Abstract | Publisher Full Text | Free Full Text
Mc Cord KA, Ewald $\mathrm{H}$, Ladanie $\mathrm{A}$, et al:: Current use and costs of electronic health records for clinical trial research: a descriptive study. CMAJ open. 2019; 7(1): E23-E32.

PubMed Abstract | Publisher Full Text | Free Full Text

McKay A, Jones A, Gamble C, et al:: Data sets used and Supplementary Table 1. figshare. Dataset. 2020.

http://www.doi.org/10.6084/m9.figshare.12185193.v1 


\section{Open Peer Review}

\section{Current Peer Review Status: ?}

\section{Version 1}

Reviewer Report 13 May 2020

https://doi.org/10.5256/f1000research.25738.r63052

(C) 2020 Love S. This is an open access peer review report distributed under the terms of the Creative Commons Attribution License, which permits unrestricted use, distribution, and reproduction in any medium, provided the original work is properly cited.

\section{Sharon Love \\ MRC Clinical Trials Unit, University College London, London, UK}

This is a review of the protocols of RCTs, currently in progress, funded by NIHR, UK. RCTs were selected from NIHR HTA funding stream list if they claimed to be using routinely collected data for at least one study outcome. The authors found 102 trial protocols matching this criteria and from data extraction that 46 of these were using routinely collected data solely for at least one outcome. The research also found that a handful referenced previous feasibility work confirming the quality of the EHR and also gives a useful table categorising for the 102 trials how they used EHR.

\section{Major Comments}

I have only one major comment and it is the reason for both the 'partly' options below. The sample was selected to be using routinely collected data for at least one study outcome. Therefore I think the main result should contain this information. I consider that "in a UK cohort" is not enough of a description of the cohort. The fact that the sample was selected based on using routine data for an outcome is crucial in the interpretation.

The main result is that of 102 protocols using routinely collected data for an outcome, 46 were using routinely collected data as their sole source for at least one outcome. $46 / 102=45 \%$. Around a half of NIHR HTA funded trials that had an uploaded protocol and used routinely collected health data for at least one study outcome, used solely routinely collected data for at least one trial outcome.

I think this is an important result.

\section{Minor comments}

1. Abstract - last part of the last sentence has a word missing "The majority of which were carried out in North America".

2. If you have space in the text, it would be useful to add the information that 30 were omitted due to not having a protocol. 
3. The flow chart shows you selected the papers by selecting RCT, those that had a protocol and then those using routinely collected data for at least one outcome. I would be tempted to list the inclusion criteria in the paper in the same order.

4. The second inclusion criteria is "use of routinely collected health data". Elsewhere you use the term EHR. I would be tempted to be consistent.

5. Table 1: category 10 description appears incomplete.

6. Table 1: could you add a footnote of the definition of a registry trial?

Is the work clearly and accurately presented and does it cite the current literature? Yes

Is the study design appropriate and is the work technically sound?

Yes

Are sufficient details of methods and analysis provided to allow replication by others? Yes

If applicable, is the statistical analysis and its interpretation appropriate?

Partly

Are all the source data underlying the results available to ensure full reproducibility? Yes

Are the conclusions drawn adequately supported by the results? Partly

Competing Interests: With others, I have conducted a review of the use of routinely collected health data by using release lists from registries which has been accepted for publication but is not yet published.

Reviewer Expertise: Trial conduct, particularly monitoring and the use of routinely collected health data.

I confirm that I have read this submission and believe that I have an appropriate level of expertise to confirm that it is of an acceptable scientific standard, however I have significant reservations, as outlined above.

Author Response 19 May 2020

Andrew McKay

Major comments: Thanks for your important comments. We have made these clearer within the article update to version 2. 
Major comments part 1: We have now made it clear that the "UK cohort" is "NIHR HTA trials with a protocol" ongoing at the stated data extraction date.

Major comments part 2: We have now made this clearer.

Minor comments: Thank you for your comments. We have addressed them all within the article update to version 2. In relation to one specific comment, we have chosen to use 'routinely collected health data (RCHD)' throughout rather than 'Electronic Health Record (EHR)' for consistency.

Competing Interests: None.

The benefits of publishing with F1000Research:

- Your article is published within days, with no editorial bias

- You can publish traditional articles, null/negative results, case reports, data notes and more

- The peer review process is transparent and collaborative

- Your article is indexed in PubMed after passing peer review

- Dedicated customer support at every stage

For pre-submission enquiries, contact research@f1000.com 